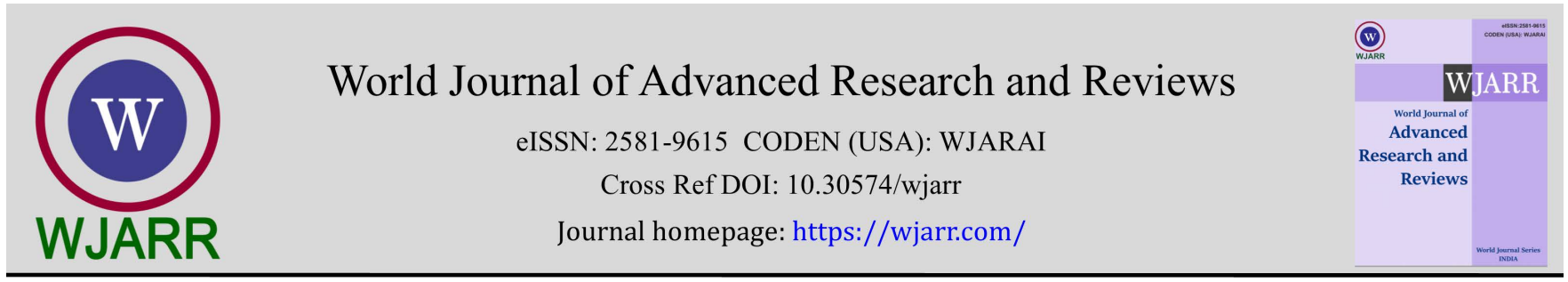

(REVIEW ARTICLE)

\title{
The potency of PLGA-Encapsulated Epigallocatechin Gallate (EGCG) as adjuvant therapy for chronic kidney disease
}

\author{
Deandra Maharani Widiatmaja ${ }^{1}$, Alyaa Ulaa Dhiya Ul Haq ${ }^{1}$, Dinda Dwi Purwati ${ }^{1}$ and Arifa Mustika 2,* \\ ${ }_{1}^{1}$ Medical Program, Faculty of Medicine, Universitas Airlangga, Surabaya, Indonesia. \\ 2 Department of Anatomy, Histology, and Pharmacology, Faculty of Medicine, Universitas Airlangga, Surabaya, Indonesia.
}

World Journal of Advanced Research and Reviews, 2022, 13(01), 273-279

Publication history: Received on 06 December 2021; revised on 09 January 2022; accepted on 11 January 2022

Article DOI: https://doi.org/10.30574/wjarr.2022.13.1.0019

\begin{abstract}
Chronic kidney disease (CKD) prevalence keeps increasing worldwide and being particular concern due to its morbidity and mortality. However, current CKD therapy are known to be economically costly and not necessarily provide better outcomes. Epigallocatechin gallate (EGCG) is one of the substances that widely studied as perspective therapeutic agents of CKD due to its anti-inflammatory, antioxidant, and enhancing mitochondrial function ability. However, the use of EGCG is limited to low bioavailability and poor pharmacokinetic profile. Encapsulation of EGCG with PLGA is expected to increase the efficacy of EGCG especially for its use as the kidney protective agent and optimize therapy of CKD. Thus, this study aims to analyze the potency of PLGA-encapsulated EGCG as the adjuvant therapy for CKD. This study was a narrative review summarizes studies related to current adjuvant therapy of CKD. EGCG has beneficial effects in reducing pro-inflammatory cytokines among chronic kidney disease. EGCG also can increase scavenging of free radicals to decrease reactive oxygen species. EGCG is known to enhance mitochondrial function and increase mitochondrial protection to prevent apoptosis in various kidney diseases. Combination of PLGA encapsulation with EGCG has a beneficial effect in improving the delivery, bioavailability, stability, and the pharmacokinetic profile of EGCG. PLGAencapsulated EGCG also provides a better therapeutic effect on preventing and decreasing progression of kidney damage. Finally, this study concluded that combination of PLGA-encapsulated EGCG has a potency as the adjuvant therapy of CKD.
\end{abstract}

Keywords: CKD; EGCG; Kidney; Nanoparticles; PLGA; Therapy

\section{Introduction}

Chronic kidney disease (CKD) is condition of an abnormality in kidney structure or function lasting more than 3 months, with specific implications including GFR of less than $60 \mathrm{~mL} / \mathrm{min} / 1.73 \mathrm{~m} 2$ or the occurrence of $\geq 1$ signs of kidney dysfunction such as albuminuria [1]. In 2017, CKD was reported to occur in $9.1 \%$ of population worldwide or estimated 697.5 million people suffer from CKD [2]. In Indonesia, the prevalence of CKD keeps increasing annually from $20 \%$ in 2013 to $38 \%$ in 2018 with the highest proportion in the range of $65-74$ years (82.3\%) [3]. Several metabolic changes such as diabetes and obesity are contributing to the development of CKD, mainly through increase of reactive oxygen species (ROS) production and elevate inflammatory processes which later affect the cellular function [4,5] Prolonged exposure to ROS could induce oxidative stress and accelerate progression of kidney damage so that increases the risk of morbidity and mortality [6]. Nowadays, dialysis combined with drugs and lifestyle changes are still being the standard treatment for CKD. However, dialysis and current CKD therapy are known to be economically costly and not necessarily provide better outcomes [7]. Hemodialysis (HD) was found to increase and exacerbate oxidative stress in CKD patients, due not only to a lack of exogenous antioxidant intake, but also to increase antioxidant molecule loss and oxidative products accumulation [8] Increased formation of powerful pro-oxidant reactive molecules in HD also induce

\footnotetext{
${ }^{*}$ Corresponding author: Arifa Mustika

Department of Anatomy, Histology, and Pharmacology, Faculty of Medicine, Universitas Airlangga, Surabaya, Indonesia. 
complement and inflammatory pathway activation [9]. This process later contributes to the onset of systemic cardiovascular disease [8]. Thus, the provision of adjuvant therapy is widely being developed and being of special concern in order to increase the effectiveness of CKD therapy.

Nowadays, catechins as one of the natural polyphenolic molecules have started to be studied as therapeutic agents against metabolic changes and oxidative stress. One included in the catechins family is Epigallocatechin gallate (EGCG) $(\mathrm{Mw}=458,372 \mathrm{~g} / \mathrm{mol})$, which is the most abundant polyphenol contained (60-65\%) in green tea (Camelia sinensis) [10] [11], that is considerably to have benefit in CKD therapy. Several studies have shown that EGCG has a strong antioxidant and anti-inflammatory effect with great efficacy in reducing kidney damage. It was observed that EGCG also has high safety and very small adverse effects, which can then be a promising treatment for CKD [4,12-14] Click or tap here to enter text.

However, the implementation of EGCG in clinical use is still limited due to low stability, poor absorption, and low bioavailability. Only about $0.1-1.1 \%$ of the dose reaches systemic circulation from orally administered EGCG from natural sources $[5,14]$. This evidence has become a trigger to increase delivery efficiency of EGCG to enhance its therapeutic effect. One perspective is the application of nanotechnology, specifically EGCG-loaded nanoparticles. Polylactide-co-glycolic acid (PLGA) is one of nanoparticles composed of a polymeric base that is known to have ability in increasing drug stability and bioavailability [5,15]. Encapsulation of EGCG with PLGA is expected to increase the efficacy of EGCG especially for its use as the kidney protective agent and optimize therapy of CKD [5]. Therefore, this review aims to analyze the potency of PLGA-encapsulated EGCG as the adjuvant therapy for CKD.

\section{Method}

This study was a narrative review done by searching, collecting, and analyzing studies related to current adjuvant therapy for chronic kidney disease. Literature search was conducted in several databases such as "PubMed", "Science Direct", and government official websites, following these relevant keywords: "EGCG", "PLGA", "nanoparticles", "therapy", “CKD", "kidney". The authors used "Mesh Database" and Boolean Operator ("AND", "OR", "NOT") to get specific search results. The literature search was limited to the last 10 years published studies and available in English or Indonesian.

\section{Results and discussion}

\subsection{Pathogenesis of Chronic Kidney Disease}

The development of CKD is determined by several factors. Chronic damage in the kidney is greatly influenced by the oxidative stress (OS), which is defined as an imbalance between excessive oxidants (free radicals) and their degradation by an insufficient antioxidant system. Reactive oxygen species (ROS) and reactive nitrogen species (RNS) are oxidant components that are actually formed under physiological conditions and can be removed by auto-antioxidant defense mechanisms [16]. However, ROS production in cells may be increased with environmental and lifestyle triggers like exposure to cigarette smoke, UV radiation, heavy metal ions, insecticides and so on [17]. Even more, the kidney is a highly metabolic organ and rich in oxidation reaction in mitochondria so it is more susceptible to OS. ROS can inhibit cellular activity also damage cellular proteins, lipids, and nucleic acids $[16,18]$. Long-term OS exposure can accelerate kidney cortex and medulla damage, as well as induce broad effects such as impairment of kidney blood flow, sodium and fluid over-retention, inflammation, decrease kidney function, even of causing fibrotic changes and onset of proteinuria. Increased ROS production is also an independent factor of CKD progression to the advanced stage. These mechanisms can contribute to the occurrence of CKD complication, such as cardiovascular disease, mineral bone disease, and gastrointestinal disorder [16,19]

Another important mechanism that plays a crucial role in the progression of CKD is inflammation response [20]. Chronic inflammatory state in CKD is contributed by numerous factors, such as acidosis, chronic and recurrent infections, intestinal dysbiosis and altered adipose tissue metabolism. Several major pro-inflammatory cytokines including interleukin-6 (IL-6), interleukin-1 (IL-1), tumor necrosis factor- $\alpha$ (TNF- $\alpha$ ), which are produced by adipose tissue and have been positively associated with the severity of CKD. Apart from being an independent process, increased proinflammatory cytokines also occur as a response to prolonged oxidative stress processes. Other [21].

Mitochondrial dysfunction has an important role in the progression of CKD. Studies reveal persistent disruption of the mitochondrial structure and sustained tubular damage in acute kidney injury can lead to gradual progression of CKD [22]. As mentioned before, long-term exposure to oxidative stress also affects the mitochondrial function and increases 
mitophagy. Stressor and inflammation also can disrupt the mitochondrial biogenesis (MB), known as the process of forming mitochondrial organelles, along with mtDNA in them through the proliferation of previous organelles [22,23]. Impaired MB can lead to maladaptive repair of microlesion and further can develop into fibrosis progression [23,24]. Increased kidney damage will result in worsen kidney function and increase the oxidative stress, inflammation, as well as the mitochondrial dysfunction. These three main mechanisms determining CKD progression also contribute to the onset of systemic complications such as cardiovascular disease and cancer [25-27]

\subsection{EGCG Mechanism of Action as a Renoprotection}

Epigallocatechin gallate (EGCG) is one of the major catechins present in green tea [10] In a review said A cup of green tea typically brewed from $2.5 \mathrm{~g}$ of tea leaves contains 240-320 mg of catechins, of which EGCG accounts for 60-65\% [11]. EGCG has many characteristics such as antioxidant, anti-inflammatory, and anti-apoptotic, which of these characteristics has been proven to treat or prevent for various kidney diseases [4]. Thus, it is expected that these several functions would be beneficial in the treatment of chronic kidney disease (CKD).

\subsubsection{Anti-inflammatory effect of EGCG}

EGCG has been shown to be anti-inflammatory by suppressing the synthesis and action of inflammatory pathway such as, peroxynitrite, nitric oxide synthase, reactive oxygen species, and also cyclooxygenase-2 [28]. On the other study, EGCG inhibited the levels of pro-inflammatory cytokines including TNF- $\alpha$, IL-1 $\beta$, and IL-6 [29]. EGCG can significantly reduce inflammation in the kidney, as evidenced by a decreases MPO and IL-1 $\beta$ activity [30]. Administration of EGCG as a treatment can normalize pro-inflammatory cytokine levels in unilateral ureteral obstruction that result in increased kidney function. As a result, EGCG is thought to contribute to enhanced kidney function as renoprotection by acting as an antioxidant and anti-inflammatory. The modulation of NF-kB and Nrf2 is involved in this renoprotection [31]. EGCG administration significantly suppressed the increase in pro-inflammatory cytokines in serum and kidney tissue, as indicated by lower levels of TNF-, IL-6 and IL-1 in the EGCG group [32].

\subsubsection{Antioxidant Effect of EGCG}

Several studies show that EGCG has a strong antioxidant effect. EGCG has antioxidant activity through a variety of mechanisms, such as catalytic metal chelation, hydrogen atom transfer, and electron transfer. Furthermore, in a way that relates to chemical, the antioxidant activity of EGCG can be explained by the presence of the polyhydroxyl structure and the gallate group, which both play important roles in scavenging of free radicals, as well as the presence of phenolic groups that are easily oxidized, resulting in the formation of a quinone [33]. Administration of EGCG affects the oxidative stress parameters [34]. Compared to other polyphenols, EGCG is the most effective against H2O2-induced HT22 cell stress and has a high potential to reduce ROS generation and radical scavenging [35]. EGCG can significantly reduce oxidative stress and inflammation in the kidney, as evidenced by a decrease in malondialdehyde (MDA) levels, and an increase in SOD activity. EGCG also decreases MPO and IL-1 $\beta$ activity [30]. EGCG activates the Nrf2-ARE and PPARr pathways and attenuates the AKT/ERK/NF-kB pathway to reduce oxidative stress and activates SIRT1 to facilitate tissue repair and regeneration [36].

\subsubsection{EGCG Enhance Mitochondrial Function}

The administration of EGCG in CKD is known to have beneficial properties in preventing mitochondrial dysfunction. EGCG could prevent endoplasmic reticulum (ES) stress by involving NF- $\varkappa$ B and Nrf2 signaling pathways. EGCG significantly decreases NF- $\varkappa$ B and enhance Nrf2 activity thus prevent mitochondrial apoptosis related to ER stress [37]. EGCG also increases antioxidant production in mitochondrial cell such as SOD, CAT, GPX, c-GST, glutamate cysteine ligase, and heme oxygenase-1 (HO-1) [38]. so that enhance increases self-defense to ER stress. In addition, EGCG provides mitochondrial protection by increasing the mitochondrial electron chain complex and enhancing the MnSOD and GPX enzyme function [39]. EGCG has beneficial effects in enhancing mitochondrial function by regulating mitochondrial metabolism, including mitochondrial biogenesis, mitochondrial bioenergy, and the cell cycle as well as apoptosis. EGCG also reduced the inhibition of mitochondrial respiration, and conserved the NADH pool leading to high ATP synthesis [40]. EGCG can also prevent kidney damage and kidney fibrosis in cadmium-induced chronic kidney injury model [12] and protect against cisplatin-induced nephrotoxicity by suppressing apoptosis in mouse kidney tubular epithelial cells [41].

\subsection{PLGA Encapsulation Improve Bioavailability and Efficacy of EGCG}

Epigallocatechin gallate (EGCG) has multiple benefits and targets mechanisms of action that match the signaling pathway of CKD pathogenesis. In addition, EGCG also has other advantages including low toxicity, less cost, and easy accessibility [42]. However, the use of EGCG as an adjuvant therapy in clinical practice is still limited due to its low 
bioavailability. EGCG is known to have low chemical stability, high degradation in intestinal environment, and low intestinal absorbance [5,14]. In oral administration, EGCG will be exposed to enzyme activities and variable pH range. Despite having good resistance to acidic gastric $\mathrm{pH}$, EGCG is unstable to alkaline pH in the intestine [43]. Free EGCG also has a shorter half-life and high clearance rate. Thus, these mechanisms contribute to decrease bioavailability and stability, and have also been a concern regarding the impact on EGCG efficacy $[5,43]$

The use of nanoparticles as EGCG carrier molecules is currently being developed because apart from increasing the bioavailability of EGCG, studies have shown that nanoencapsulation can also increase the efficacy and therapeutic effects of EGCG [44]. Nowadays, polylactide-co-glycolic acid (PLGA) is one of the nanoparticles that is widely used as a carrier molecule of EGCG. PLGA is a polymer-based nanoparticle composed of a circular structure of nanometer polymeric size, in which the drug is distributed evenly in the matrix [5,15]. PLGA is suitable for water-soluble purposes and known to have unique physical properties such as higher tissue permeability and colloidal stability. Compared to other types of nanoparticles, PLGA is an FDA-approved polymer with great biocompatibility and biodegradability. PLGA-encapsulated EGCG preparation is also more simple and more economic so that it becomes favourable to use in recent studies $[5,15,45]$

Several studies have shown that PLGA could significantly modify the pharmacokinetic profile and increases the bioavailability of EGCG both in vitro and in vivo $[5,42,46,47]$. It was also known that PLGA-encapsulated EGCG has high encapsulation efficiency and drug loading efficiency [5,47]. Encapsulation with PLGA causes a sustained release of EGCG and avoids gross degradation due to exposure of changed $\mathrm{pH}$ in the digestive environment $[5,42,47]$. In addition to promoting better chemical stability, the administration of EGCG-loaded PLGA-NPs both intravenous and oral route also showed a significant increase in bioavailability of EGCG in the blood [42,47]. which in oral administration the bioavailability increased up to 2.4-fold compared to free EGCG [5]. PLGA provides higher bioavailability through increasing half-life and decreasing clearance rate of EGCG [5].

Encapsulation by PLGA presents a higher tissue permeability so that it can increase the absorption of EGCG in the intestinal wall $[5,46]$ when passing through gastric acid, surface erosion and hydrolytic cleavage of the PLGA matrix still occurs. However, due to higher resistance of PLGA to acidic $\mathrm{pH}$, smaller bioproducts which are encapsulated-PLGA drugs will be formed and reduce in these particles' size contribute to the enhancement of drug penetration [46]. PLGAencapsulated EGCG also provides the possibility for better antioxidant effects, marked by effective ROS scavenging in $[47,48]$.

A study using a nephrotic syndrome model shows that the administration of oral PLGA-encapsulated EGCG can reduce kidney damage and prevent chronic progression of glomerular disease. PLGA-encapsulated EGCG therapy is shown to have better efficacy in reducing proteinuria excretion and kidney pathology scores to certain degrees, compared to free EGCG [5]. In addition, PLGA-encapsulated EGCG has been known to enhance antitumor effect and DNA repair [42,46,47]. which can also make this regimen as a chemo-preventive therapy for complications of CKD, such as kidney fibrosis or kidney tumor. The current evidence shows that the addition of PLGA can optimize the role of EGCG and these compound combinations could be a perspective as CKD adjuvant therapy. Further studies both pre-clinical and clinical studies, are needed to ascertain the benefits and safety of this combination, especially for CKD therapy.

\section{Conclusion}

In conclusion, the use of PLGA-encapsulated EGCG has a potency as the CKD adjuvant therapy. EGCG has several mechanisms of action that can inhibit the signaling pathway of CKD, such as anti-inflammatory, antioxidant, and enhancing mitochondrial function. PLGA nanoparticles encapsulation has a beneficial effect in improving the delivery, bioavailability, stability, and the pharmacokinetic profile of EGCG. Therefore, the PLGA encapsulation also can enhance the therapeutic effect of EGCG. PLGA-encapsulated EGCG also provides better efficacy in reducing and preventing progression of kidney damage. This study supports further examination including pre-clinical and clinical studies to evaluate benefits and safety of this combination for adjuvant therapy for CKD patients.

\section{Compliance with ethical standards}

\section{Acknowledgments}

The authors would like to thanks all the parties who helped in writing of this review article. 


\section{Disclosure of conflict of interest}

The authors state no conflict of interest.

\section{References}

[1] Charles C, Ferris AH. Chronic Kidney Disease. Primary Care - Clinics in Office Practice. 2020; 47: 585-95.

[2] Bikbov B, Purcell CA, Levey AS, Smith M, Abdoli A, Abebe M, et al. Global, regional, and national burden of chronic kidney disease, 1990-2017: a systematic analysis for the Global Burden of Disease Study 2017. The Lancet. 2020; 395: 709-33.

[3] Indonesian Health Ministry. Hasil Riset Kesehatan Dasar Kementerian Kesehatan RI. 2018.

[4] Kanlaya R, Thongboonkerd V. Molecular Mechanisms of Epigallocatechin-3-Gallate for Prevention of Chronic Kidney Disease and Renal Fibrosis: Preclinical Evidence. Current Developments in Nutrition. $2019 ; 3$.

[5] Zhang G, Zhang J. Enhanced oral bioavailability of EGCG using pH-sensitive polymeric nanoparticles: Characterization and in vivo investigation on nephrotic syndrome rats. Drug Design, Development and Therapy. 2018; 12: 2509-18.

[6] Lackner RP, Lanuti M, Leal TA, Leisch LJ, Lilenbaum R, Lin J, et al. Clinical Practice Guidelines in Oncology NCCN Categories of Evidence and Consensus. 2017; 15.

[7] Breyer MD, Susztak K. Developing Treatments for Chronic Kidney Disease in the 21st Century. Seminars in Nephrology. 2016; 36: 436-47.

[8] Rysz J, Franczyk B, Ławiński J, Gluba-Brzózka A. Oxidative stress in esrd patients on dialysis and the risk of cardiovascular diseases. Antioxidants. 2020; 9: 1-24.

[9] Liakopoulos V, Roumeliotis S, Gorny X, Dounousi E, Mertens PR. Oxidative Stress in Hemodialysis Patients: A Review of the Literature. Oxidative Medicine and Cellular Longevity. 2017.

[10] Li F, Wang Y, Li D, Chen Y, Qiao X, Fardous R, et al. Perspectives on the recent developments with green tea polyphenols in drug discovery. Expert Opinion on Drug Discovery. 2018; 13: 643-60.

[11] Hayakawa S, Ohishi T, Miyoshi N, Oishi Y, Nakamura Y, Isemura M. Anti-cancer effects of green tea epigallocatchin-3- gallate and coffee chlorogenic acid. Molecules. 2020; 25.

[12] Zhou P, Yu JF, Zhao CG, Sui FX, Teng X, Wu Y bin. Therapeutic potential of EGCG on acute renal damage in a rat model of obstructive nephropathy. Molecular Medicine Reports. 2013; 7: 1096-102.

[13] Chen CY, Kao CL, Liu CM. The cancer prevention, anti-inflammatory and anti-oxidation of bioactive phytochemicals targeting the TLR4 signaling pathway. International Journal of Molecular Sciences. 2018 ; 19.

[14] Krupkova O, Ferguson SJ, Wuertz-Kozak K. Stability of (-)-epigallocatechin gallate and its activity in liquid formulations and delivery systems. Journal of Nutritional Biochemistry. 2016; 37: 1-12.

[15] Yang XH, Zhang BL, Zhang XM, Tong JD, Gu YH, Guo LL, et al. EGCG Attenuates Renal Damage via Reversing Klotho Hypermethylation in Diabetic db/db Mice and HK-2 Cells. Oxidative Medicine and Cellular Longevity. 2020.

[16] Daenen K, Andries A, Mekahli D, van Schepdael A, Jouret F, Bammens B. Oxidative stress in chronic kidney disease. Pediatric Nephrology. 2019; 34: 975-91.

[17] Sharifi-Rad M, Anil Kumar N v., Zucca P, Varoni EM, Dini L, Panzarini E, et al. Lifestyle, Oxidative Stress, and Antioxidants: Back and Forth in the Pathophysiology of Chronic Diseases. Frontiers in Physiology. $2020 ; 11$.

[18] Che R, Yuan Y, Huang S, Zhang A. Mitochondrial dysfunction in the pathophysiology of renal diseases. Am J Physiol Renal Physiol. 2014; 306: 367-78.

[19] Drozdz D, Kwinta P, Sztefko K, Kordon Z, Drozdz T, Łątka M, et al. Oxidative stress biomarkers and left ventricular hypertrophy in children with chronic kidney disease. Oxidative Medicine and Cellular Longevity 2016.

[20] Qian Q. Inflammation: A Key Contributor to the Genesis and Progression of Chronic Kidney Disease. Contributions to Nephrology. 2017; 191: 72-83.

[21] Rapa SF, di Iorio BR, Campiglia P, Heidland A, Marzocco S. Inflammation and oxidative stress in chronic kidney disease-potential therapeutic role of minerals, vitamins and plant-derived metabolites. International Journal of Molecular Sciences. 2020; 21. 
[22] Takemura K, Nishi H, Inagi R. Mitochondrial Dysfunction in Kidney Disease and Uremic Sarcopenia. Frontiers in Physiology. 2020; 11.

[23] Jiang M, Bai M, Lei J, Xie Y, Xu S, Jia Z, et al. Mitochondrial dysfunction and the AKI-to-CKD transition. American Journal of Physiology - Renal Physiology. 2020; 319: F1105-16.

[24] Panizo S, Martínez-Arias L, Alonso-Montes C, Cannata P, Martín-Carro B, Fernández-Martín JL, et al. Fibrosis in chronic kidney disease: Pathogenesis and consequences. International Journal of Molecular Sciences. 2021; 22: 1-19.

[25] Agharazii M, St-Louis R, Gautier-Bastien A, Ung RV, Mokas S, Larivière R, et al. Inflammatory cytokines and reactive oxygen species as mediators of chronic kidney disease-related vascular calcification. American Journal of Hypertension. 2015; 28: 746-55.

[26] Lowrance WT, Ordoñez J, Udaltsova N, Russo P, Go AS. CKD and the risk of incident cancer. Journal of the American Society of Nephrology. 2014; 25: 2327-34.

[27] Wong G, Staplin N, Emberson J, Baigent C, Turner R, Chalmers J, et al. Chronic kidney disease and the risk of cancer: An individual patient data meta-analysis of 32,057 participants from six prospective studies. BMC Cancer. 2016; 16.

[28] Hagiu A, Attin T, Schmidlin PR, Ramenzoni LL. Dose-dependent green tea effect on decrease of inflammation in human oral gingival epithelial keratinocytes: in vitro study. 2019.

[29] Pervin M, Unno K, Ohishi T, Tanabe H, Miyoshi N, Nakamura Y. Beneficial Effects of Green Tea Catechins on Neurodegenerative Diseases. Molecules. 2018; 23.

[30] Tain YL, Hsu CN. Targeting on asymmetric Dimethylarginine-Related nitric oxide-reactive oxygen species imbalance to reprogram the development of hypertension. International Journal of Molecular Sciences. 2016; 17.

[31] Wang Y, Wang B, Du F, Su X, Sun G, Zhou G, et al. Epigallocatechin-3-Gallate Attenuates Oxidative Stress and Inflammation in Obstructive Nephropathy via NF- $\mathrm{BB}$ and Nrf2/HO-1 Signalling Pathway Regulation. Basic and Clinical Pharmacology and Toxicology. 2015; 117: 164-72.

[32] Lv J, Feng M, Zhang LL, Wan X, Zeng YC, Liang PF, et al. Protective effect of epigallocatechin gallate, a major constituent of green tea, against renal ischemia-reperfusion injury in rats. International Urology and Nephrology. 2015; 47: 1429-35.

[33] Shirakami Y, Shimizu M. Possible mechanisms of green tea and its constituents against cancer. Molecules 2018; 23.

[34] Bulboaca AE, Boarescu PM, Porfire AS, Dogaru G, Barbalata C, Valeanu M, et al. The effect of nanoepigallocatechin-gallate on oxidative stress and matrix metalloproteinases in experimental diabetes mellitus. Antioxidants. 2020; 9.

[35] He J, Xu L, Yang L, Wang X. Epigallocatechin gallate is the most effective catechin against antioxidant stress via hydrogen peroxide and radical scavenging activity. Medical Science Monitor. 2018; 24: 8198-206.

[36] Ye T, Zhen J, Du Y, Zhou JK, Peng A, Vaziri ND, et al. Green tea polyphenol (-)-epigallocatechin-3-gallate restores Nrf2 activity and ameliorates crescentic glomerulonephritis. PLoS ONE. 2015; 10.

[37] Bao H, Peng A. The Green Tea Polyphenol(-)-epigallocatechin-3-gallate and its beneficial roles in chronic kidney disease. Journal of Translational Internal Medicine. 2016; 4: 99-103.

[38] Shi W, Li L, Ding Y, Yang K, Chen Z, Fan X, et al. The critical role of epigallocatechin gallate in regulating mitochondrial metabolism. Future Medicinal Chemistry. 2018; 10: 795-809.

[39] Pan H, Chen J, Shen K, Wang X, Wang P, Fu G, et al. Mitochondrial modulation by epigallocatechin 3 -gallate ameliorates cisplatin induced renal injury through decreasing oxidative/nitrative stress, inflammation and NFkB in mice. PLoS ONE. 2015; 10.

[40] Yu NH, Pei H, Huang YP, Li YF. (-)-epigallocatechin-3-gallate inhibits arsenic-induced inflammation and apoptosis through suppression of oxidative stress in mice. Cellular Physiology and Biochemistry. 2017; 41: 1788-800.

[41] Chen B, Liu G, Zou P, Li X, Hao Q, Jiang B, et al. Epigallocatechin-3-gallate protects against cisplatin-induced nephrotoxicity by inhibiting endoplasmic reticulum stress-induced apoptosis. Experimental Biology and Medicine. 2015; 240: 1513-9. 
[42] Kazi J, Sen R, Ganguly S, Jha T, Ganguly S, Chatterjee Debnath M. Folate decorated epigallocatechin-3-gallate (EGCG) loaded PLGA nanoparticles; in-vitro and in-vivo targeting efficacy against MDA-MB-231 tumor xenograft. International Journal of Pharmaceutics. 2020; 585.

[43] Fangueiro JF, Parra A, Silva AM, Egea MA, Souto EB, Garcia ML, et al. Validation of a high performance liquid chromatography method for the stabilization of epigallocatechin gallate. International Journal of Pharmaceutics. 2014; 475: 181-90.

[44] Yang QQ, Wei XL, Fang YP, Gan RY, Wang M, Ge YY, et al. Nanochemoprevention with therapeutic benefits: An updated review focused on epigallocatechin gallate delivery. Critical Reviews in Food Science and Nutrition. 2020; 60: 1243-64.

[45] Safer AM, Leporatti S, Jose J, Soliman MS. Conjugation of EGCG and chitosan NPS as a novel nano-drug delivery system. International Journal of Nanomedicine. 2019; 14: 8033-46.

[46] Cano A, Ettcheto M, Chang JH, Barroso E, Espina M, Kühne BA, et al. Dual-drug loaded nanoparticles of Epigallocatechin-3-gallate (EGCG)/Ascorbic acid enhance therapeutic efficacy of EGCG in a APPswe/PS1dE9 Alzheimer's disease mice model. Journal of Controlled Release. 2019; 301: 62-75.

[47] Zhang L, Chen W, Tu G, Chen X, Lu Y, Wu L, et al. Enhanced chemotherapeutic efficacy of plga-encapsulated epigallocatechin gallate (EGCG) against human lung cancer. International Journal of Nanomedicine. 2020; 15: 4417-29.

[48] Shin YC, Yang WJ, Lee JH, Oh JW, Kim TW, Park JC, et al. PLGA nanofiber membranes loaded with epigallocatechin3-0-gallate are beneficial to prevention of postsurgical adhesions. International Journal of Nanomedicine. 2014; 9: 4067-78. 